Journal of Mathematics and Statistics 2 (1): 346-350, 2006

ISSN 1549-3644

(C) 2006 Science Publications

\title{
The Solution of Heat Conduction Equation with Mixed Boundary Conditions
}

\author{
Naser Abdelrazaq \\ Department of Basic and Applied Sciences, Tafila Technical University \\ P.O. Box 179, Tafila, Jordan
}

\begin{abstract}
The study is devoted to determine a solution for a non-stationary heat equation in axial symmetric cylindrical coordinates under mixed discontinuous boundary of the first and second kind conditions, with the aid of a Laplace transform and separation of variables method used to solve the considered problem which is the dual integral equations method.
\end{abstract}

Key words: Nonstationary heat equation, dual integral equations, mixed boundary conditions

\section{INTRODUCTION}

The method of dual integral equations is widely used to solve elliptic partial differential equation with many physical and technical applications ${ }^{[1-4]}$, also several techniques were developed in the last fifty years to solve dual equations with different coordinate systems. discuss In this study the solution of twodimensional non-stationary heat conduction problem in axially symmetrical cylindrical coordinates with discontinuous mixed boundary conditions first and second kind on the level surface of a semi-infinite solid cylindrical coordinates will be discussed. The solution of the problem is based on the application of dual integral equations method with the help of the Laplace transform and separation of variables. It is known that the solution of dual integral equations introduced to some type of singular integral equations of the first kind with unknown function, weight and free term depend on the parameter of a Laplace transform. The exact solution of such integral equation can be obtained by expressing its unknown function in the form of a functional series in powers of a Laplace transform parameter The main goal of given problem in this work is to extend the use of dual integral equations method to solve parabolic partial differential equations with mixed discontinuous boundary conditions, by using some discontinuous integrals technique. This technique is applied to solve different type of dual equations related to diffraction theory, elasticity theory and other application ${ }^{[3,4]}$, when the second one of a dual integral equations is homogeneous. In heat conduction theory, there are several methods were used for solving heat conduction problems under unmixed boundary condition as pointed out in ${ }^{[5-7]}$. Mandrik reduced some dual equations to the Fredholm integral equation of the second kind ${ }^{[8,9]}$.

\section{MATHEMATICAL FORMULATION OF THE PROBLEM}

The main aim of this study is to solve the nonstationary heat conductivity differential equation for a half-space in cylindrical coordinates with axially symmetry

$\frac{\partial^{2} T}{\partial r^{2}}+\frac{1}{r} \frac{\partial T}{\partial r}+\frac{\partial^{2} T}{\partial z^{2}}=\frac{1}{a} \frac{\partial T}{\partial \tau}$

where $T=T(r, z, \tau)$ is the temperature distribution function $, 0<r<\infty, 0<z<\infty$ are the corresponding cylindrical coordinates, $\tau>0$ is a time, $a \neq 0$ is the temperature conductivity coefficient (constant). The equation (2.1) can be solved by using the conditions

$\left.\frac{\partial T}{\partial r}\right|_{r=0}=\left.\frac{\partial T}{\partial r}\right|_{r \rightarrow \infty}=\left.T\right|_{z \rightarrow \infty}=0$

under mixed discontinuous boundary conditions first and second kind on the level surface $z=0$

$T(r, 0, \tau)=f_{1}(r, \tau), r \in S$,

$\partial T(r, 0, \tau) / \partial z=f_{2}(r, \tau), \quad r \in \bar{S}$,

where $S=(0, R) \quad \bar{S}=(R, \infty)$. The initial condition is

$T(r, z, 0)=0$.

The unknown functions $f_{1}, f_{2}$ in (2.3),(2.4) continuous and have the limited variation with respect of each variables $r$ and $\tau$, moreover

$\int_{0}^{\infty}\left|f_{i}(r, \tau)\right| d r<\infty \int_{0}^{\infty}\left|f_{i}(r, \tau)\right| d \tau<\infty, i=1,2$.

These restrictions allow to apply Laplace transform with respect to $\tau$ and Hankle transform with respect to $\mathrm{r}$ moreover, we assume that the functions $f_{i}(r, \tau), i=1,2$ have absolutely continuous derivative with respect to $r$. The physical significance of the given boundary value problem is that, inside the disk $0<r<R$, on $z=0$, the temperature function is given by $T(r, 0, \tau)=f_{1}(r, \tau)$ and outside the disk $R<r<\infty$ at $z=0$ a heat flow is given by $T_{z}(r, 0, \tau)=f_{2}(r, \tau)$. Next, to simplify the investigation of the solution, we will assume ideal insulation exist, i.e, $f_{2}=0$.

Corresponding Author: $\quad$ Dr. Naser Abdelrazaq, Department of Basic and Applied Sciences, Tafila Technical University, P.O. Box 179, Tel: 9622 0777489652, Tafila-Jordan, Fax: 2250033, 


\section{SOLUTION OF THE PROBLEM}

The boundary-value problem mentioned above should be solved by applying of the Laplace transform in the $\tau$-variable. Defining of $\bar{T}(r, z, s)$ as ${ }^{[10]}$

$\bar{T}(r, z, s)=\int_{0}^{\infty} T(r, z, \tau) \exp (-s \tau) d \tau$

Taking the Laplace transform and separation of variables to equation (2.1) with regard to conditions (2.2), the general solution of the problem is obtained in form of improper integral

$\bar{T}(r, z, s)=\int_{0}^{\infty} \bar{u}(p, s) \exp \left(-\sqrt{p^{2}+\alpha}\right) J_{0}(p r) d p$

where $J_{0}(p r)$ is the Bessel function of the first kind of order zero, $p$ is the parameter of separation of variables, $\mathrm{s}$ is the parameter of Laplace transform with $\alpha=s / a \operatorname{Re}(s)>0$.

Applying the Laplace transform to the boundary conditions (2.3),(2.4) and using these conditions to the general solution (3.1), we obtain the following dual integral equations to determine the unknown function $u(p, s)$

$\int_{0}^{\infty} \bar{u}(p, s) J_{0}(p r) d p=\bar{f}_{1}(r, s), r \in S$

$\int_{0}^{\infty} \bar{u}(p, s) \sqrt{p^{2}+\alpha} J_{0}(p r) d p=\overline{f_{2}}(r, s), r \in \bar{S}$

In general equations (3.2), (3.3) can be solved by using discontinuous integral technique, if the second equation is homogeneous, i.e, $\bar{f}_{2}(r, s)=0$, thus to simplify the investigation of the solution, we will assume ideal insulation exist, i.e $f_{2}=0$.In general when the second dual integral equations is non-homogenous, it can be reduced to the homogeneous by expansion $\bar{f}_{2}(r, s)$ in Hankle's integral transform

$$
\bar{f}_{2}(r, s)=\int_{0}^{\infty} \bar{F}(p, s) \sqrt{p^{2}+\alpha} J_{0}(p r) d p,
$$

Where $\bar{F}(p, s)$ is known function determine by the inversion Hankle transform

$$
\bar{F}(p, s)=\frac{p}{\sqrt{p^{2}+s / a}} \int_{R}^{\infty} y \bar{f}_{2}(y, s) J_{0}(p y) d y .
$$

Next write down $\bar{u}(p, s)-\bar{F}(p, s)=\bar{A}(p, s)$, then use the expansion (3.4), the following dual integral equations are obtained to determine the unknown function $\bar{A}(p, s)$

$$
\begin{aligned}
& \int_{0}^{\infty} \bar{A}(p, s) J_{0}(p r) d p=\bar{D}(r, s), \quad r \in S \\
& \int_{0}^{\infty} \bar{A}(p, s) \sqrt{p^{2}+s / a} J_{0}(p r) d p=0, r \in \bar{S}
\end{aligned}
$$

where

$$
\bar{D}(r, s)=\bar{f}_{1}(r, s)-\int_{0}^{\infty} \bar{F}(p, s) J_{0}(p r) d p
$$

As $s \rightarrow 0$, the dual integral equations (3.2) and (3.3) tend to the dual integral equations of the form

$$
\begin{aligned}
& \int_{0}^{\infty} u(p) J_{0}(p r) d p=f_{1}(r), \quad r \in S, \\
& \int_{0}^{\infty} p u(p) J_{0}(p r) d p=f_{2}(r), r \in \bar{S} .
\end{aligned}
$$

More detail, discussed in monographs ${ }^{[1,3]}$

To simplify the solution of equations (3.2),(3.3), , we will consider special cases such that $\bar{f}_{2}(r, s)=0, \bar{f}_{1}(r, s)=f(r) / s$, thus, the dual equations $(3,2),(3,3)$ will take the form

$$
\begin{aligned}
& \int_{0}^{\infty} \bar{u}(p, s) J_{0}(p r) d p=f(r) / s, \quad r \in S \\
& \int_{0}^{\infty} \bar{u}(p, s) \sqrt{p^{2}+\alpha} J_{0}(p r) d p=0, r \in \bar{S}
\end{aligned}
$$

Replacing the function $\bar{u}(p, s)$ by another unknown function $\bar{\sigma}(t, s)$ with the help of the relation

$$
\bar{u}(p, s)=\frac{p}{\sqrt{p^{2}+\alpha}} \int_{0}^{R} \bar{\sigma}(t, s) \cos \left(t \sqrt{p^{2}+\alpha}\right) d t .
$$

It is assumed $\bar{\sigma}(t, s)$ is differentiable with respect to $\mathrm{t}$, where $t \in(0, R)$, also the inverse Laplace transform $L^{-1}[\bar{\sigma}(t, s)]=\sigma(t, \tau)$ exist., further, we assume that $\sigma(t, \tau)$ is continuous or piecewise continuous in any interval $\tau_{1}<\tau<\tau_{2}$ for $\tau_{1}>0$ and $\tau^{n}|\sigma(t, \tau)|$ is bounded as $\tau \rightarrow 0^{+}$, moreover $\sigma(t, \tau)$ is exponential order namely $\exp (-\gamma \tau)|\sigma(t, \tau)|$ is bounded for some positive number $\gamma$ as $\tau \rightarrow \infty^{[5]}$.

Substituting (3.7) into (3.6) and integrating with respect to $t$ from 0 to $R$, then interchanging the order of integration, using the value of the discontinuous integral $^{[11,12]}$

$$
\begin{aligned}
& \int_{0}^{\infty} \frac{p J_{0}(p r)}{\sqrt{p^{2}+\alpha}} \sin \left(x \sqrt{p^{2}+\alpha}\right) d p \\
& =\left\{\begin{array}{cc}
0, & r>x, \\
\frac{\cos \sqrt{\left(x^{2}-r^{2}\right) \alpha}}{\sqrt{x^{2}-r^{2}}}, & x>r,
\end{array}\right.
\end{aligned}
$$

ensure the equality (3.6) to zero, such that $\left(\left(\sigma_{t}(t, s)=\partial \sigma(t, s) / \partial t\right)\right.$

$$
\begin{aligned}
& \bar{\sigma}(R, s)\left(\int_{0}^{\infty} \frac{p J_{0}(p r)}{\sqrt{p^{2}+s / a}} \sin \left(R \sqrt{p^{2}+\alpha}\right) d p\right) \\
& -\int_{0}^{R} \overline{\sigma_{t}^{\prime}}(t, s)\left(\int_{0}^{\infty} \frac{p J_{0}(p r)}{\sqrt{p^{2}+\alpha}} \sin \left(t \sqrt{p^{2}+\alpha}\right) d p\right) d t=0
\end{aligned}
$$


Substituting (3.7) into (3.5) and using the discontinuous integral ${ }^{[12]}$

$$
\begin{aligned}
& \int_{0}^{\infty} \frac{p J_{0}(p r)}{\sqrt{p^{2}+\alpha}} \cos \left(t \sqrt{p^{2}+\alpha}\right) d p \\
& = \begin{cases}\frac{-1}{\sqrt{t^{2}-r^{2}}} \sin \sqrt{\left(t^{2}-r^{2}\right) \alpha} & 0<r<t<R, \\
\frac{1}{\sqrt{r^{2}-t^{2}}} \exp \left(-\sqrt{\left(r^{2}-t^{2}\right) \alpha}\right) & 0<t<r<R .\end{cases}
\end{aligned}
$$

A first kind singular integral equation is obtained to determine the unknown function $\bar{\sigma}(t, s)$

$$
\begin{aligned}
& \int_{0}^{r} \frac{\bar{\sigma}(t, s)}{\sqrt{r^{2}-t^{2}}} \exp \left(-\sqrt{\left(r^{2}-t^{2}\right) s / a}\right) d t \\
& -\int_{r}^{R} \frac{\bar{\sigma}(t, s)}{\sqrt{t^{2}-r^{2}}} \sin \sqrt{\left(t^{2}-r^{2}\right) s / a} d t=f(r) / s, r \in S .
\end{aligned}
$$

Since $\bar{\sigma}(t, s)$ is analytical function of a parameter $\mathrm{s}$, it can represent a functional series in power of degree $s^{1 / 2}$

$$
\bar{\sigma}(t, s)=\exp (-R \sqrt{\alpha}) \sum_{n=0}^{\infty} \sigma_{n}(t) s^{n / 2-1}
$$

The inverse Laplace transform for (3.9) exist ${ }^{[8]}$

$L^{-1} \bar{\sigma}(t, s)=\sigma(t, \tau)=\frac{2}{\sqrt{\pi}} \exp \left(-L^{2}\right) \sum_{n=0}^{\infty} \frac{H_{n-1}(L)}{2^{n} \tau^{n / 2}} \sigma_{n}(t)$,

$$
L^{2}=\frac{R^{2}}{4 a \tau}
$$

$H_{n-1}(L)$ is a Hermite function ${ }^{[13]}$. Multiplying equation (3.8) by $\exp (R \sqrt{\alpha})$ and expanding $\exp (-x)$ and $\sin (x)$ in (3.8) in appropriate Maclaurin series, then using expression (3.9) ,the following integral equation is obtained to determine the unknown members $\sigma_{0}(t), \sigma_{1}(t), \sigma_{2}(t), \ldots$

$\sum_{n=0}^{\infty} \sum_{m=0}^{\infty} \frac{(-1)^{m} s^{\frac{m+n}{2}}}{m !(\sqrt{a})^{m}} \int_{0}^{r} \sigma_{n}(t)\left(r^{2}-t^{2}\right)^{\frac{m-1}{2}} d t-$

$\sum_{n=0}^{\infty} \sum_{m=0}^{\infty} \frac{(-1)^{m} s^{\frac{n+1}{2}+m}}{(2 m+1) !(\sqrt{a})^{2 m+1}} \int_{r}^{R} \sigma_{n}(t)\left(t^{2}-r^{2}\right)^{m} d t$

$=\sum_{l=0}^{\infty} \frac{1}{l !}\left(\frac{R}{\sqrt{a}}\right)^{l} s^{l / 2} f(r)$

Equating the coefficient of the left and right hand sides in (3.10) as equal powers of $s^{1 / 2}$ we find that At $s^{0}: l=0 ;\{(\mathrm{m}, \mathrm{n})=(0,0)\}$, we get an Abels integral equation to evaluate $\sigma_{0}(t)$

$\int_{0}^{r} \frac{\sigma_{0}(t)}{\sqrt{r^{2}-t^{2}}} d t=f(r)$

Solving Abels integral equations (3.11) yields $\sigma_{0}(t)=\frac{2}{\pi} \frac{d}{d t} \int_{0}^{r} \frac{y f(y)}{\sqrt{r^{2}-y^{2}}} d y$

At $s^{1 / 2}: l=1 ;\{(\mathrm{m}, \mathrm{n})=(1,0),(0,1),(0,0)\}$, an Abels integral equation obtained to determine $\sigma_{1}(t)$ $\int_{0}^{r} \frac{\sigma_{1}(t)}{\sqrt{r^{2}-t^{2}}} d t-\frac{1}{1 ! \sqrt{a}} \int_{0}^{R} \sigma_{0}(t) d t=f(r) \frac{R}{! \sqrt{a}}$

Using the inversion for determining $\sigma_{1}(t)$ in (3.12), so that the solution is given by

$\sigma_{1}(t)=\frac{2}{\pi} \frac{d}{d t} \int_{0}^{t} \frac{y}{\sqrt{t^{2}-y^{2}}}\left\{f(y) \frac{R}{\sqrt{a} 1 !}+\frac{1}{1 ! \sqrt{a}} \int_{0}^{R} \sigma_{0}(\xi) d \xi\right\} d y$

At $s^{1}: l=2, \quad\{(\mathrm{~m}, \mathrm{n})=(0,2),(2,0),(1,1),(0,1)\}$, in similar process as in (3.11) and (3.12) the solution for $\sigma_{2}(t)$ is given by

$\sigma_{2}(t)=\frac{2}{\pi} \frac{d}{d t} \int_{0}^{t} \frac{y}{\sqrt{t^{2}-y^{2}}}$

$\left\{f(y) \frac{R^{2}}{(\sqrt{a})^{2} 2 !}-\frac{(-1)^{2}}{2 !(\sqrt{a})^{2}} \int_{0}^{y} \sigma_{0}(\xi) \sqrt{y^{2}-\xi^{2}} d \xi+\frac{1}{1 ! \sqrt{a}} \int_{0}^{R} \sigma_{1}(\xi) d \xi\right\} d y$

From the above evaluation of $\sigma_{0}(t), \sigma_{1}(t), \sigma_{2}(t), \ldots$. it is easy to conclude that $\sigma_{n}(t)$ satisfy the following recurrent formula, for even index $\mathrm{n}$

$\int_{0}^{r} \frac{\sigma_{n}(t)}{\sqrt{r^{2}-t^{2}}} d t+\sum_{k=1}^{[n / 2]} \frac{(-1)^{n-2(k-1)}}{(n-2(k-1)) !(\sqrt{a})^{(n-2(k-1))}}$

$\int_{0}^{r} \sigma_{2(k-1)}(t)\left(\sqrt{r^{2}-t^{2}}\right)^{n-(2 k-1)} d t$

$-\sum_{k=1}^{[n / 2]} \frac{(-1)^{n / 2-k}}{(n-(2 k-1)) !(\sqrt{a})^{n-(2 k-1)}}$

$\int_{0}^{R} \sigma_{(2 k-1)}(t)\left(\sqrt{t^{2}-r^{2}}\right)^{n-2 k} d t=f(y) \frac{R^{n}}{(\sqrt{a})^{n} n !}$

The solution Abel integral equation (3.13) for the unknown function $\sigma_{n}(t)$ in terms of the known functions $\sigma_{2 k-1}(t), \sigma_{2(k-1)}(t)$ is given by:

$\sigma_{n}(t)=\frac{2}{\pi} \frac{d}{d t} \int_{0}^{t} \frac{y}{\sqrt{t^{2}-y^{2}}}$

$\left\{\begin{array}{c}f(y) \frac{R^{n}}{(\sqrt{a})^{n} n !}+ \\ \sum_{k=1}^{[n / 2]} \frac{(-1)^{n / 2-k}}{(n-(2 k-1)) !(\sqrt{a})^{n-(2 k-1)}} \int_{0}^{R} \sigma_{(2 k-1)}(\xi)\left(\sqrt{\xi^{2}-y^{2}}\right)^{n-2 k} d \xi- \\ \sum_{k=1}^{[n / 2]} \frac{(-1)^{n-2(k-1)}}{(n-2(k-1)) !(\sqrt{a})^{n-2(k-1)}} \int_{0}^{y} \sigma_{2(k-1)}(\xi)\left(\sqrt{y^{2}-\xi^{2}}\right)^{n-(2 k-1)} d \xi\end{array}\right\}$

On the other hand for odd values of $n$ we have the recurrent formula

$\int_{0}^{r} \frac{\sigma_{n}(t)}{\sqrt{r^{2}-t^{2}}} d t$

$+\sum_{k=1}^{\left\lfloor\frac{n-1}{2}\right\rfloor} \frac{(-1)^{n-(2 k-1)}}{(n-(2 k-1)) !(\sqrt{a})^{n-(2 k-1)}} \int_{0}^{r} \sigma_{(2 k-1)}(t)\left(\sqrt{r^{2}-t^{2}}\right)^{n-2 k} d t$

$-\sum_{k=1}^{\left\lceil\frac{n-1}{2}\right]} \frac{(-1)^{k}}{(2 k+1) !(\sqrt{a})^{(2 k+1)}} \int_{0}^{R} \sigma_{n-(2 k+1)}(t)\left(\sqrt{t^{2}-r^{2}}\right)^{2 k} d t$

$=f(y) \frac{R^{n}}{(\sqrt{a})^{n} n !}$, 
Again treating (3.15) as an Abels integral equation to determine $\sigma_{n}(t)$ for odd index $\mathrm{n}$

$$
\begin{aligned}
& \sigma_{n}(t)=\frac{2}{\pi} \frac{d}{d t} \int_{0}^{t} \frac{y}{\sqrt{t^{2}-y^{2}}} \\
& \left\{\begin{array}{l}
f(y) \frac{R^{n}}{(\sqrt{a})^{n} n !}+ \\
\sum_{k=1}^{n-1-1} \frac{(-1)^{k}}{(2 k+1) !(\sqrt{a})^{(2 k+1)} \int_{0}^{R} \sigma_{n-(2 k+1)}(\xi)\left(\sqrt{\xi^{2}-y^{2}}\right)^{2 k} d \xi-} \\
\sum_{k=1}^{n-1} \frac{(-1)^{n-(2 k-1)}}{(n-(2 k-1)) !(\sqrt{a})^{n-(2 k-1)}} \int_{0}^{y} \sigma_{(2 k-1)}(\xi)\left(\sqrt{y^{2}-\xi^{2}}\right)^{n-2 k} d \xi
\end{array}\right\} d y
\end{aligned}
$$

In particular ,if the temperature function inside the region $0<\mathrm{r}<\mathrm{R}, \mathrm{z}=0$ is constant, say $\bar{f}(r, s)=K / s$ in equation (3.10) then the value of each $\sigma_{i}(t), i=1, \ldots, n$ is polynomial in t.

$$
\begin{aligned}
& \sigma_{0}(t)=\frac{2}{\pi} K \\
& \sigma_{1}(t)=\frac{2}{\pi} K \frac{R}{\sqrt{a}}\left(1+\frac{2}{\pi}\right) \\
& \sigma_{2}(t)=\frac{2}{\pi} \frac{1}{2 !} \frac{R^{2}}{(\sqrt{a})^{2}}+\frac{1}{1 !(\sqrt{a})}\left(\frac{2}{\pi}\right)^{2}\left(1+\frac{2}{\pi}\right)-\frac{1}{2 !(\sqrt{a})^{2}} K\left(\frac{2}{\pi}\right) t^{2}
\end{aligned}
$$

In general if the function $\bar{f}(r, s)$ depends on time(in general case), it should be expressed as $\bar{f}(r, s)=\sum_{j=0}^{\infty} f_{j}(r) s^{j / 2}$,

hence, the right hand side of (3.10) can be written as $\sum_{l=0}^{\infty} \sum_{j=0}^{\infty} \frac{1}{l !}\left(\frac{R}{\sqrt{a}}\right)^{l} f_{j}(r) s^{(l+j) / 2}$.

Equating the left and the right hand side of (3.10) to determine recurrence relations to $\sigma_{n}(t)$ for even and odd index $\mathrm{n}$ in powers of $s^{(l+j) / 2}$

To determine the general solution $\bar{T}(r, z, s)$, substituting equations (3.14) and (3.16) into equation (3.9), then putting the obtaining result into (3.7) and finally substituting the result into the general solution (3.1) we get

$$
\begin{aligned}
& \bar{T}(r, z, s)=\exp (-R \sqrt{\alpha}) \sum_{n=0}^{\infty} s^{n / 2} \\
& \int_{0}^{R} \sigma_{n}(t)\left(\int_{0}^{\infty} \frac{p J_{0}(p r)}{\sqrt{p^{2}+\alpha}} \cos \left(t \sqrt{p^{2}+\alpha}\right) \exp \left(-z \sqrt{p^{2}+\alpha}\right) d p\right) d t .(3.17) \\
& \text { Now express in }
\end{aligned}
$$
$(\exp (i x)+\exp (-i x)) / 2, i=\sqrt{-1}$ and use the value of the integral $^{[12]}$

$$
\int_{0}^{\infty} x \frac{\exp \left(-a \sqrt{x^{2}+y^{2}}\right)}{\sqrt{x^{2}+y^{2}}} J_{0}(c x) d x=\frac{\exp \left(-y \sqrt{a^{2}+c^{2}}\right)}{\sqrt{a^{2}+c^{2}}}
$$

The solution of (3.17) with regard to (3.17-a) can be written as

$$
\begin{aligned}
& \bar{T}(r, z, s)=\frac{1}{2} \exp (-R \sqrt{\alpha}) \sum_{n=0}^{\infty} s^{n / 2} \\
& \int_{0}^{R} \sigma_{n}(t)\left\{\frac{\exp \left(-R_{1} \sqrt{\alpha}\right)}{R_{1}}+\frac{\exp \left(-R_{2} \sqrt{\alpha}\right.}{R_{2}}\right\} d t .
\end{aligned}
$$

where $R_{1}^{2}=(z-i t)^{2}+r^{2}, R_{2}^{2}=(z+i t)^{2}+r^{2}$. It is clear that as $\mathrm{r}$ and $\mathrm{z}$ tend to infinity $\bar{T}(r, z, s)$ is vanished. The inverse Laplace transform of (3.18) is

$$
\begin{aligned}
& T(r, z, \tau)=\frac{1}{2 \sqrt{\pi}} \sum_{n=0}^{\infty} \int_{0}^{R} \frac{\sigma_{n}(t)}{2^{n} \tau^{n / 2}} \\
& \left\{\exp \left(-L_{1}^{2}\right) H_{n-1}\left(L_{1}\right)+\exp \left(-L_{2}^{2}\right) H_{n-1}\left(L_{2}\right)\right\} d t \\
& \text { where } L_{1}^{2}=\frac{\left(R+R_{1}\right)^{2}}{4 a \tau}, L_{2}^{2}=\frac{\left(R+R_{2}\right)^{2}}{4 a \tau} .
\end{aligned}
$$

Introduction the solution of dual integral equations (3.5) and (3.6) to integral equation of the second kind: We will discuss another technique for solving the above dual equations (3.5) and (3.6) by reducing these equations to second kind singular integral equations. Rewriting equation (3.8) in the following form using the relation $\operatorname{ch}(x)-\operatorname{sh}(x)=\exp (-x)$

$$
\begin{aligned}
& \int_{0}^{r} \bar{\sigma}(t, s) \frac{c h \sqrt{\left(r^{2}-t^{2}\right) s / a}}{\sqrt{r^{2}-t^{2}}} d t \\
& -\int_{0}^{R} \bar{\sigma}(t, s) \frac{\sin \sqrt{\left(t^{2}-r^{2}\right) s / a}}{\sqrt{t^{2}-r^{2}}} d t=\bar{f}(r, s), r \in S
\end{aligned}
$$

The next step is expanding in (4.1) $\operatorname{ch}(x), \operatorname{sh}(x)$, in appropriate Maclurin series, then rearranging the obtaining result, asimple calculation yields a first kind integral equation to determine the unknown function $\bar{\sigma}(t, s)$

$$
\begin{aligned}
& \int_{0}^{r} \frac{\bar{\sigma}(t, s)}{\sqrt{r^{2}-t^{2}}} d t+\sum_{m=1}^{\infty} \frac{s^{m}}{a^{m}(2 m) !} \int_{0}^{r} \bar{\sigma}(t, s)\left(r^{2}-t^{2}\right)^{m-1 / 2} d t- \\
& \sum_{m=0}^{\infty} \frac{(-1)^{m} s^{m+1 / 2}}{a^{m+1 / 2}(2 m+1) !} \int_{0}^{R} \bar{\sigma}(t, s)\left(t^{2}-r^{2}\right)^{m} d t=\bar{f}(r, s), r \in S
\end{aligned}
$$

Treating (4.2) as an Ables' integral equation by applying the inversion for $\bar{\sigma}(t, s)$, then interchanging the order of integration inside the integral sign for the obtaining result, a second kind singular integral equation is achieved as

$$
\begin{aligned}
& \bar{\sigma}(t, s)=\bar{F}(t, s)-\int_{0}^{t} \bar{\sigma}(\xi, s) \bar{N}(\xi, t, s) d \xi \\
& +\int_{0}^{R} \bar{\sigma}(\xi, s) \bar{M}(\xi, t, s) d \xi
\end{aligned}
$$

where $\bar{F}(t, s)=\frac{2}{\pi} \frac{d}{d t} \int_{0}^{t} \frac{y f(y)}{\sqrt{t^{2}-y^{2}}} d y$

$$
\bar{M}(\xi, t, s)=\frac{2}{\pi} \sum_{m=0}^{\infty} \frac{s^{m+1 / 2}(-1)^{m}}{(2 m+1) ! a^{m+1 / 2}} \frac{d}{d t} \int_{0}^{t} \frac{y\left(\xi^{2}-y^{2}\right)^{m}}{\sqrt{t^{2}-y^{2}}} d y,
$$


$\bar{N}(\xi, t, s)=\frac{2}{\pi} \sum_{m=1}^{\infty} \frac{s^{m}}{2 m ! a^{m}} \frac{d}{d t} \int_{\xi}^{t} \frac{y\left(y^{2}-\xi^{2}\right)^{m-1 / 2}}{\sqrt{t^{2}-y^{2}}} d y$.

Substituting the expansion (3.9), into equation (4.3) an integral equation of the second kind is obtained to evaluate the sequence $\sigma_{i}(t), i=0,1,2, \ldots \ldots, n$

$$
\begin{aligned}
& \sum_{n=0}^{\infty} \sigma_{n}(t) s^{n / 2}=\sum_{n=0}^{\infty} \frac{R^{l} s^{l / 2}}{a^{l / 2} l !} F(t)+\frac{2}{\pi} \\
& \sum_{m=0}^{\infty} \sum_{n=0}^{\infty} \frac{s^{\frac{2 m+n+1}{2}}(-1)^{m}}{(2 m+1) ! a^{m+1 / 2}} \int_{0}^{R} \sigma_{n}(t) M_{m}(t, \xi) d \xi \\
& -\frac{2}{\pi} \sum_{m=1}^{\infty} \sum_{n=0}^{\infty} \frac{s^{\frac{2 m+n}{2}}}{2 m ! a^{m}} \int_{0}^{t} \sigma_{n}(\xi) N_{m}(t, \xi) d \xi
\end{aligned}
$$

where

$$
\begin{aligned}
& M_{m}(t, \xi)=\frac{2}{\pi} \frac{d}{d t} \int_{0}^{t} \frac{y\left(\xi^{2}-y^{2}\right)^{m}}{\sqrt{t^{2}-y^{2}}} d y \\
& =\frac{2}{\pi} \frac{d}{d t} t \xi^{2 m}{ }_{2} F_{1}\left(1,-m, 3 / 2, t^{2} / \xi^{2}\right) \\
& =\frac{2}{\pi}\left(\begin{array}{c}
\xi^{2 m}{ }_{2} F_{1}\left(1,-m, 3 / 2, t^{2} / \xi^{2}\right) \\
-\frac{4 m t^{2} \xi^{2 m-2}}{3}{ }_{2} F_{1}\left(2,1-m, 5 / 2, t^{2} / \xi^{2}\right)
\end{array}\right) .
\end{aligned}
$$

Where ${ }_{2} F_{1}$ is a hypergeometric function ${ }^{[14]}$ and

$$
\begin{aligned}
& N_{m}(t, \xi)=\frac{2}{\pi} \frac{d}{d t} \int_{\xi}^{t} \frac{y\left(y^{2}-\xi^{2}\right)^{m-1 / 2}}{\sqrt{t^{2}-y^{2}}} d t \\
& =\frac{1}{\sqrt{\pi}} \frac{d}{d t} \frac{\Gamma(1 / 2+m)}{\Gamma(1+m)}\left(t^{2}-\xi^{2}\right)^{m}=\frac{2}{\sqrt{\pi}} \frac{m \Gamma(1 / 2+m)}{\Gamma(1+m)} t\left(t^{2}-\xi^{2}\right)^{m-1},
\end{aligned}
$$

$\Gamma(x)$ is a gamma function ${ }^{[14]}$.

If we equate the left and the right hand side of equation (4.4) with equal powers of $s^{1 / 2}$, the same recurrent relations to find $\sigma_{i}(t), i=0,1,2, \ldots ., n$ given in (3.14) and (3.16). The terms $\sigma_{i}(t), i=0,1,2, \ldots ., n$ must be satisfy the property

$\frac{\left\|\sigma_{n}(t)-\sigma_{n-1}(t)\right\|_{C^{1}(0, R)}}{\left\|\sigma_{n}(t)\right\|_{C^{1}(0, R)}}=\min$

\section{CONCLUSION}

With the help of known methods, the solution of non-stationary heat conduction equation under mixed boundary conditions is obtained by introducing the given problem to some type of dual integral equations were solved by using discontinuous integrals techniques and the known generating series $\bar{\sigma}(t, s)=\exp (-R \sqrt{\alpha}) \sum_{n=0}^{\infty} \sigma_{n}(t) s^{n / 2-1}$.

If the Laplace transform parameter appears in dual equations tends to zero, the solution of the considered problem introduced to the known results.
The above exact solution of the mixed boundary value problem given in form of infinite series can be used widely to solve various mixed boundary problems deal with unsteady state heat equation for example to infinite or finite cylinder, unsymmetrical cylindrical coordinates, spherical coordinates and other mixed problems.

\section{REFERENCES}

1. Mandal, B.N. and N. Mandal, 1999. Advances in Dual Integral Equations. London, CRC.

2. Chakrabarti, A. and N. Mandal, 1998. Solution of some dual integral equations. ZAMM. Z. Angew. Math. Mech., 78: 141-144.

3. Snedon, I., 1966. Mixed boundary value problems in potential theory. North. Hol. Pub. Amesterdam.

4. Fabricant,V.I., 1991. Mixed boundary value problems in potential theory and their applications engineering. Kluwer Academic Publishers.

5. Ozisik M.N., 1980. Heat Conduction. Wily and Sons, Inc.

6. Holman, J.P., 2002. Heat Transfer. McGraw-Hill Co. NewYork.

7. Kozlov, V.P. and P.A. Mandrik, 2000. Systems of integral and differential equations in L-parameter in problems of mathematical physics and identification methods of heat characteristics. BGU Pub. Minsk-Belarus.

8. Mandrik, P.A.,2001. The method of the dual integral equation for analysis of heat transfer processes. Mathematical Modeling and Analysis, 6: 280-288.

9. Mandrik, P.A., 2001. The solution of heat equation with mixed boundary conditions on a surface of isotropic half-space. Differentsalnye Uravneniya, 37: 238-241, (in Russian).

10. Cotta, R.M. (ed.), 1988. The integral transform method in thermal and fluids sciences and engineering. New York, Begel House, Inc.

11. Gradseyn, I.S. and I.M. Ryznik, 1992. Tables of integrals, series and products. Academic Press, New York.

12. Prodnicov, A.P., U. Brichcov and O.I. Marichev, 1983. Integral and Series of Special Functions. Moscow, Nawka.

13. Temme, N.M., 1996. Special functions an introduction to the classical functions of mathematical physics. Jhon Wiley, New York.

14. Rainville, E.D., 1971. Special Functions. Chelsea P. Com., New York. 